Hydroécol. Appl. (1990) 1/2, pp. 5-19

\title{
Inventaire piscicole de la retenue des Saints-Peyres (Tarn) à l'occasion d'une vidange décennale
}

\author{
Puy $\operatorname{Lim}^{(*)}$, Catherine Sabaton ${ }^{(*)}$ et Alain Belaud ${ }^{(*)}$
}

(*) Laboratoire d'Ichtyologie appliquée, Ecole Nationale Supérieure agronomique, Institut National Polytechnique de Toulouse, 145 avenue de Muret, 31076 Toulouse Cedex

(*) Electricité de France, Direction des Etudes et Recherches

Résumé. - Le site de la retenue des Saints-Peyres (211 hectares) a été choisi pour tenter un inventaire complet des populations piscicoles. La méthode a consisté à récupérer les poissons dans une pêcherie spécialement aménagée en aval du barrage, lors de la vidange décennale de la retenue.

Cette retenue abritait 5 tonnes de poissons de 9 espèces différentes dominées par les tanches (Tinca tinca L.) (6 490 individus pesant $4278 \mathrm{~kg}$ ), les chevaines (Leuciscus cephalus L.) (7 601 individus pesant $613 \mathrm{~kg}$ ) et les truites (Salmo trutta fario) (567 individus pesant $191 \mathrm{~kg}$ ).

II s'agit d'une retenue de faible productivité naturelle. Le peuplement piscicole est déséquilibré par un recrutement aléatoire. Cette retenue devrait donc faire l'objet, après remise en eau, d'une gestion adaptée.

Les observations sur la dévalaison des poissons en cours de vidange montrent des comportements différents se!on les espèces. Les tanches sont les plus faciles à récupérer vivantes dans la pêcherie; les chevaines et les truites subissent une forte mortalité.

Mots clés : Inventaire piscicole, retenues artificielles, vidange, tanche (Tinca tinca $L$ ), chevaine (Leuciscus cephalus L.), truite (Salmo trutta fario), comportement.

Abstract. - The fish populations were investigated in the artificial reservoir of SaintsPeyres (211 hectares of surface area). The fish sampling methods were adapted to capture the fishes in the fishing ground of barrage downstream part, especially fitted out, at the time of the reservoir decennial draining.

This reservoir covered five tons of fishes from nine species with the dominance of tench (Tinca tinca L.) (6 490 specimen weighing $4278 \mathrm{~kg}$ ), chub (Leuciscus cephalus L.) (7 601 fishes weighing $613 \mathrm{~kg}$ ) and trout (Salmo trutta fario) (567 fishes weighing $191 \mathrm{~kg})$.

It is about a reservoir of low natural productivity which the fish populations areunbalanced by hazardous recruitment. So, this reservoir should form part, after refilling, of a management conformed. 
The observation of fish rushing down in the course of drainage show the various behaviours according to species. Tenchs are easiest to capture alive in the fishing ground; chubs and trouts have a high mortality.

Key words : Fish sampling, artificial reservoirs, drainage, tench (Tinca tinca $L$ ), Chub (Leuciscus cephalus L.), Trout (Salmo trutta fario), behaviour.

\section{INTRODUCTION}

II existait déjà en 1972 plus de 12000 barrages de plus de $15 \mathrm{~m}$ de profondeur dans le monde (Toran et Mermel, 1973), couvrant environ $300000 \mathrm{~km}^{2}$ et retenant plus de $4000 \mathrm{~km}^{3}$ d'eau. Ces chiffres se sont encore considérablement accrus avec les utilisations de l'eau. Ces retenues créent des biotopes particuliers où s'instaure une biocénose différente de celle de la rivière (Ackermann et al., 1973; Cairns et al., 1978; Blaxter, 1985).

La gestion et l'exploitation de ces retenues par la pêche professionnelle ou sportive nécessitent l'évaluation puis le suivi des populations piscicoles dans ces milieux. Or, c'est seulement dans les réservoirs déjà exploités que le suivi des populations piscicoles peut être facilement réalisé par l'étude du rendement des pêches professionnelles (Mutenia, 1985; Bronte et Johnson, 1984) ou sportives (Lusk et Krcal, 1983). Ces études donnent l'évolution des populations piscicoles exploitées mais non la biomasse totale existante. II en est de même des méthodes d'échosondage (Guillard,1985; Gerdeaux, 1986) qui se limitent à la détection et à la localisation des gros poissons pélagiques dans les retenues.
Pour les retenues non exploitées, la connaissance des populations piscicoles passe soit par la mise au point de techniques de pêche d'échantillonnage valables, soit par un dénombrement complet de tous les poissons capturés lors d'opérations exceptionnelles de vidange. Le présent travail se propose d'explorer cette deuxième voie, dans une retenue propice à la maîtrise de cette opération.

Il a été procédé à une expérience de récupération quantitative des poissons de la retenue des Saints-Peyres, sur la rivière Arn (figure 1), à l'occasion de la vidange décennale de 1984. Cette opération avait aussi pour buts : de sauver les poissons et de les redistribuer dans d'autres cours d'eau et de connaître les populations piscicoles qui s'étaient naturellement établies. Cet inventaire devait aussi permettre de concevoir un plan de gestion halieutique favorisant les espèces intéressantes, après la remise en eau.

\section{MATERIEL ET METHODES}

\section{Le site}

La retenue des Saints-Peyres (figure 1) draîne un bassin versant de $129 \mathrm{~km}^{2}$ recevant annuellement en moyenne $1090 \mathrm{~mm}$ de précipitations. 


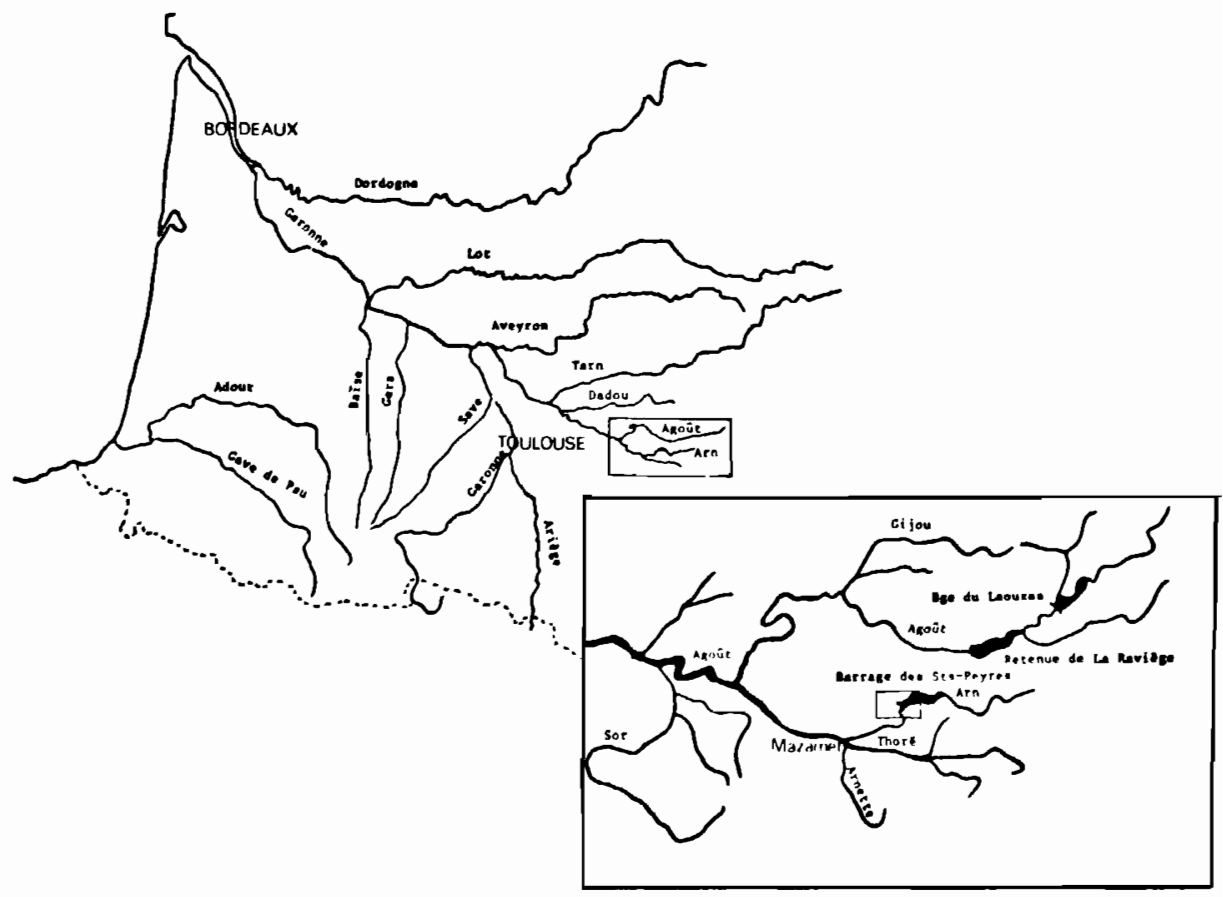

Fig. 1. - Localisation du site des Saints-Peyres.

La rivière Arn constitue le principal apport dans la retenue avec un débit moyen de l'ordre de $4,4 \mathrm{~m}^{3} / \mathrm{s}$, un maximum instantané de $272 \mathrm{~m}^{3} / \mathrm{s}$ (crue de 1953) et des étiages de $0,2 \mathrm{~m}^{3} / \mathrm{s}$. Deux ruisseaux secondaires confluent aussi dans le réservoir.

La retenue couvre 211 ha à la cote 670 NGF et retient alors $34,67 \times 10^{6} \mathrm{~m}^{3}$. Le barrage s'élève de $58,40 \mathrm{~m}$ au-dessus du thalweg. Au couronnement, sa longueur est de 175,2 m. Cette réserve d'eau est exploitée par une usine hydroélectrique, imprimant un cycle annuel avec, habituellement, des phases de remplissage en automne et surtout au printemps et des phases de surexploi- tation avec abaissement de cote en Janvier-Février d'une part et en Septembre-Octobre, d'autre part. L'amplitude des marnages annuels entre 1974 et 1983 varie entre 14 et 18,6 mètres, avec un niveau maximal en juillet et un niveau minimal en Novembre-Décembre.

La vallée est une gorge entaillée dans le gneiss. Les berges sont symétriques et très abruptes (pente moyenne de $40 \%$ près du barrage). En queue de retenue ou à la confluence des deux ruisseaux tributaires, la pente des berges est de $10 \%$ en moyenne et l'on observe un substrat de cailloux avec quelques bancs de galets, de sables et de limon. Ces plages sont transitoirement 
découvertes par les marnages, sans colonisation végétale importante (excepté pendant les assecs prolongés de la vidange). La végétation aquatique est inexistante.

Les eaux de cette retenue ont une faible minéralisation (conductibilité de l'ordre de 70 micro-Siemens $/ \mathrm{cm}$ ) et une température oscillant entre $5^{\circ} \mathrm{C}$ l'hiver et $20^{\circ} \mathrm{C}$ l'été. Les foyers de pollution en amont de la retenue sont inexistants. Les activités agricoles diffuses (prairies et élevages) renforcent la minéralisation des eaux.

Des pêches aux filets préalables à la vidange avaient permis de dresser une liste d'espèces (tanches, chevaines et truites fario principalement) et de constater, par l'étude des croissances des principales espèces, que la retenue des Saints-Peyres est un milieu oligo-mésotrophe. Les tanches présentent une structure de population déséquilibrée (insuffisance des effectifs des jeunes classes d'âge). Les truites proviennent, du moins en partie, d'opérations de repeuplement.

\section{Construction d'une pêcherie ex- périmentale}

A l'aval du barrage, il existait un accès au fond de la vallée pour atteindre le poste de commande des vannes et facilitant l'implantation d'une pêcherie (figure 2).

$\mathrm{Au}$ fond du barrage, une vanne principale ("vanne 1») débouche dans l'ancien lit sommairement aplani. Hors des périodes de crue, cette vanne du fond est fermée.
II existe une seconde voie d'évacuation des eaux constituée d'une galerie souterraine qui part de l'intérieur de la retenue, à la cote 615 NGF et qui se termine par une vanne (vanne 2, figure 2), environ 60 mètres en aval du pied du barrage. Cette vanne 2 délivre habituellement un débit réservé de $100 \mathrm{l} / \mathrm{s}$.

II a été décidé de construire la pêcherie juste en amont de la vanne 2 , de manière à ne traiter que l'eau issue de la vanne de fond (vanne 1). Cette pêcherie a été aménagée le plus en aval possible pour profiter d'une meilleure dissipation de l'énergie de l'eau (figure 2).

Le principe adopté est celui d'une grille de 9 mètres, barrant la majeure partie de la rivière entre une berge et un couloir bâti, constituant la pêcherie, où pourraient se rassembler les poissons en attendant d'être pris à l'épuisette (figure 2).

Pour faciliter les opérations de nettoyage de la grille, celle-ci a été conçue en barreaux de fer rond, de diamètre $1 \mathrm{~cm}$,et séparés par un vide de $2 \mathrm{~cm}$. La grille est installée inclinée vers l'aval de $15^{\circ}$ par rapport à la verticale. Pour éviter l'engorgement de cette grille, celle-ci a été disposée en oblique par rapport au courant. Les corps inertes tendent alors à progresser le long de la grille et, à l'extrémité, un petit dégorgeoir, permet de rassembler les débris pour les enlever plus efficacement (figure 2).

La hauteur des grilles a été fixée à 1,50 mètre, après calculs des contraintes correspondant aux débits à évacuer. 


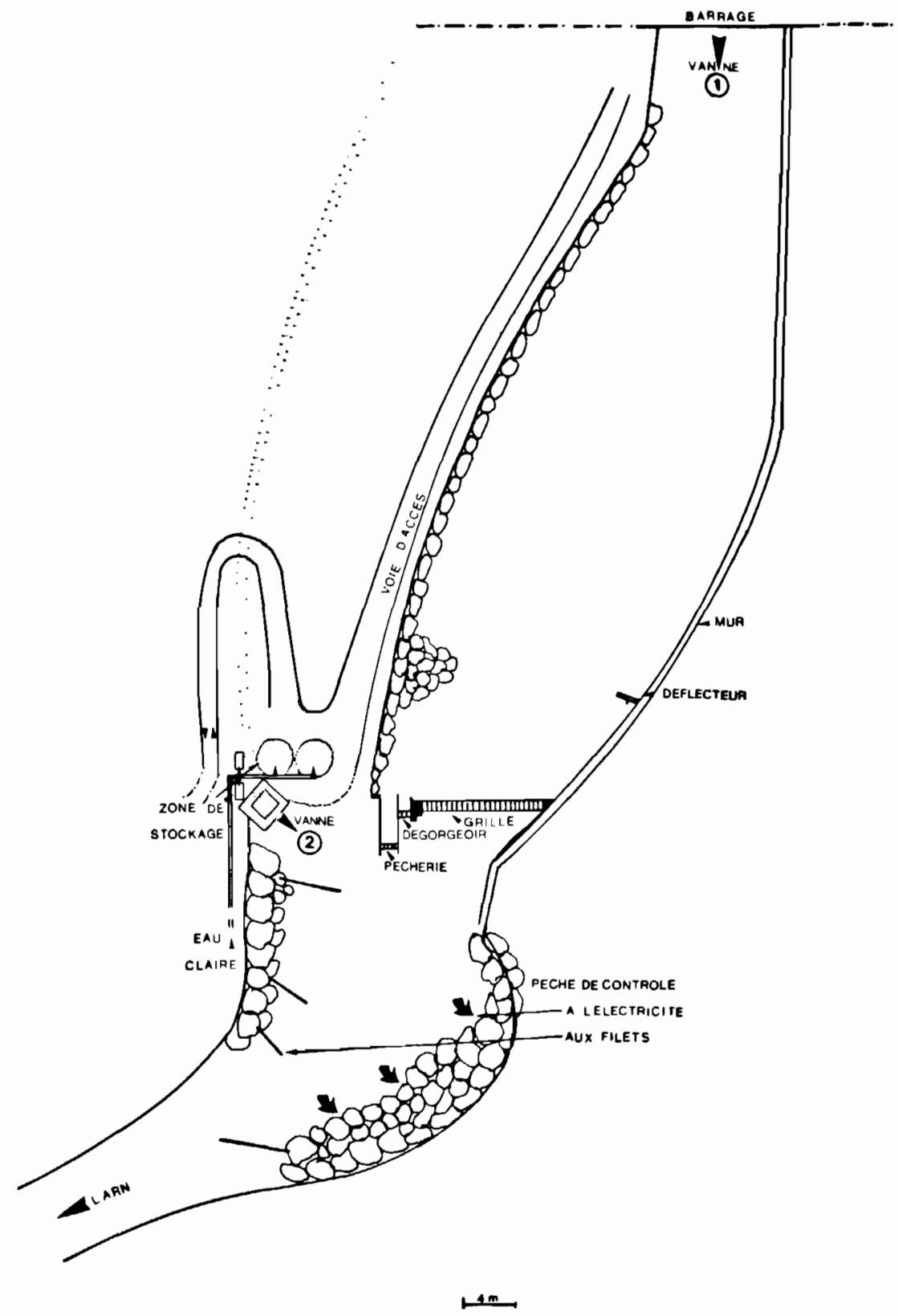

Fig. 2. - Représentation de la zone aval du barrage des Saints-Peyres, indiquant le chemin d'accès et la position de la pêcherie en aval du barrage, les zones de stockage des poissons et les sorties des vannes 1 et 2 . 
Côté couloir de pêche, une pile d'enrochements à l'amont, atténue le courant (figure 2).

\section{Adduction d'eau pour le stockage des poissons}

Pour faciliter le sauvetage des poissons, il a été monté sur place des bacs de stabulation de $12 \mathrm{~m}^{3}$, alimentés par gravité par l'eau d'un ruisseau affluent de l'Arn, capté à environ 800 mètres en aval du barrage. Le débit acheminé était de 4 litres/seconde et la température variait entre $12^{\circ} \mathrm{C}$ (la nuit) et $17^{\circ} \mathrm{C}$ en fin d'aprèsmidi.

\section{Mensurations des poissons}

Pour tous les poissons recueillis, il a été noté individuellement la longueur totale, et, si possible, le poids. En cas de trop forte affluence, les pesées de poissons vivants ont été effectuées par lots triés par espèces. Pour les tanches, le sexe a été distingué.

Les poissons survivants ont été stockés dans les bacs de stabulation, avant d'être transportés dans d'autres cours d'eau.

\section{Plan de vidange}

La procédure de vidange comprenait deux étapes :

- L'abaissement préalable du plan d'eau, commencé en février 1984, par programmation des activités des usines électriques situées en aval. A ce stade, les vannes 1 et 2 restent fermées et la vidange s'opère par les prises d'eau. L'usine a été arrêtée lorsque la cote 630 NGF a été découverte, le 20 juillet 1984 .
- Vidange du culot (environ $550000 \mathrm{~m}^{3}$ restants) par les vannes de fond, à partir du 23 juillet, à un débit de l'ordre de $5 \mathrm{~m}^{3} / \mathrm{s}$.

La cote de la retenue a évolué les 23 et 24 juillet selon la courbe de vidange représentée dans la figure 3 .

Pendant le début de la vidange du culot, par la vanne 2, des filets et des sondages par pêche électrique ont permis de repérer l'instant de la dévalaison des premiers poissons. Dès lors, la vidange a été poursuivie par ouverture de la vanne 1 et la pêcherie a été mise en service. La cote était alors à 624,80 NGF.

Pour que l'inventaire soit le plus exhaustif possible, deux barrages rustiques de $80 \mathrm{~cm}$ de dénivellés avaient été préalablement aménagés en queue de retenue. Les poissons pouvant échapper au travers des grilles de la pêcherie ont été estimés par des sondages de pêche au filet ou de pêche électrique effectués régulièrement à l'aval immédiat de la pêcherie. Après vidange, il a été procédé à une évaluation des poissons échoués, à la recherche d'éventuels poissons envasés et enfin à une pêche électrique dans le lit mineur découvert.

\section{RESULTATS}

\section{Observations générales en cours de vidange}

Les premières dévalaisons (4 truites) ont été notées à 0 h 15, le 24 juillet, pour une cote de 624,80 NGF. Elles correspondaient à des manœuvres de vannes pour évacuer des bouchons 


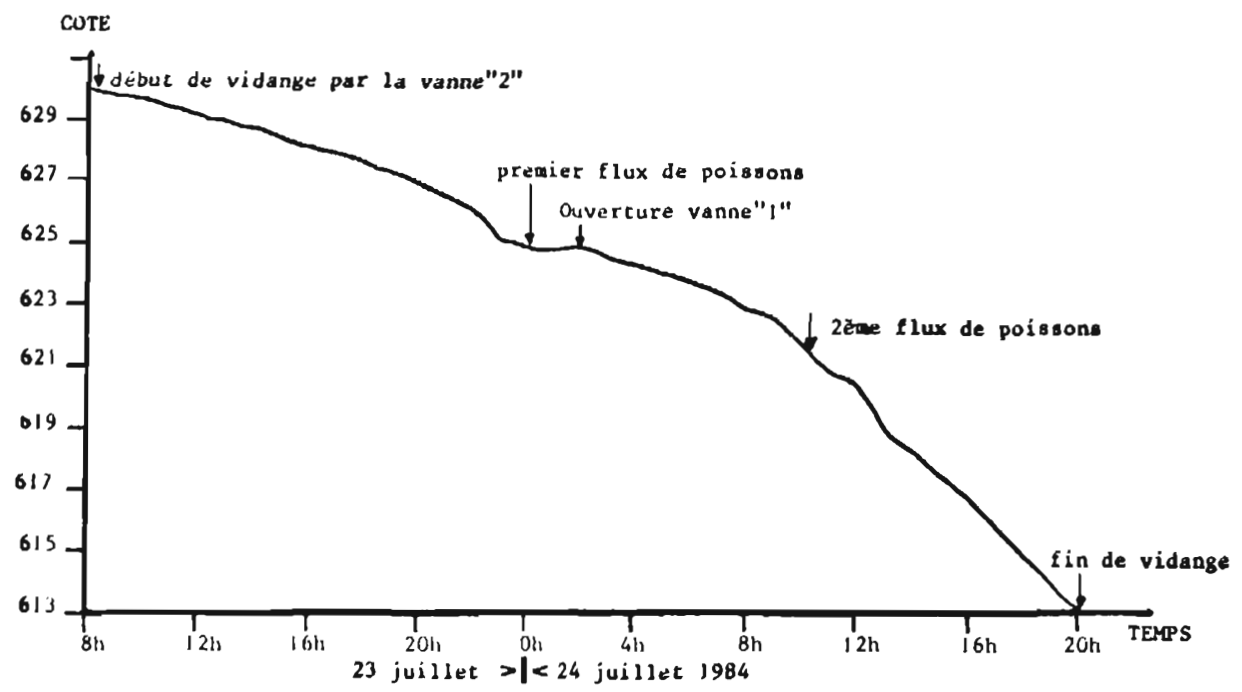

Flg. 3. - Courbe de vidange du culot de la retenue des Saints-Peyres.

vaseux formés dans la galerie (figure 2). Dès lors, il a été décidé de fermer la vanne 2 et d'ouvrir la vanne 1. La pêche de récupération commença à 2 heures. Entre temps, les pêches en aval ont permis d'évaluer à une centaine de truites $(33 \mathrm{~kg})$ et à une centaine de tanches $(63 \mathrm{~kg})$ le premier passage. A l'ouverture de la "vanne 1", il a été recueilli des truites et quelques tanches, en excellent état, ainsi que des goujons et des chevaines qui présentaient, par contre, un état de détresse.

Après ces premiers afflux, l'eau est redevenue transparente et les prises ont baissé et, entre 5 et 7 heures, on ne capturait que quelques poissons isolés. Les truites récupérées vivantes ont cessé de passer après 4 h 30.

Au cours de la matinée du 24 juillet, les captures ont repris et, à partir de 10 h 30 , l'eau est devenue défini- tivement trouble et chargée de feuilles. Les conditions de récupération se sont alors détériorées.

L'après-midi, le flot de poissons sortant par la vanne de vidange est devenu impressionnant et, malgré le renforcement des équipes de pêche et d'évacuation des bacs de poissons, il a fallu, par quatre fois, interrompre la vidange pour dégager les grilles.

En fin de vidange (à partir de $17 \mathrm{~h}$ ), la densité de poissons était telle qu'il était possible de recueillir, en tout lieu dans le plan d'eau amont de la pêcherie, des poissons très affaiblis et dérivant dans les courants de retour. Cette affuence croissante n'a cessé qu'en fin de vidange, à 20 heures.

\section{Résultats des captures}

Les poissons capturés à la pêcherie appartiennent à 9 espèces. Par ordre d'abondance, on rencontre les tanches (Tinca tinca L.) (6 149 indi- 


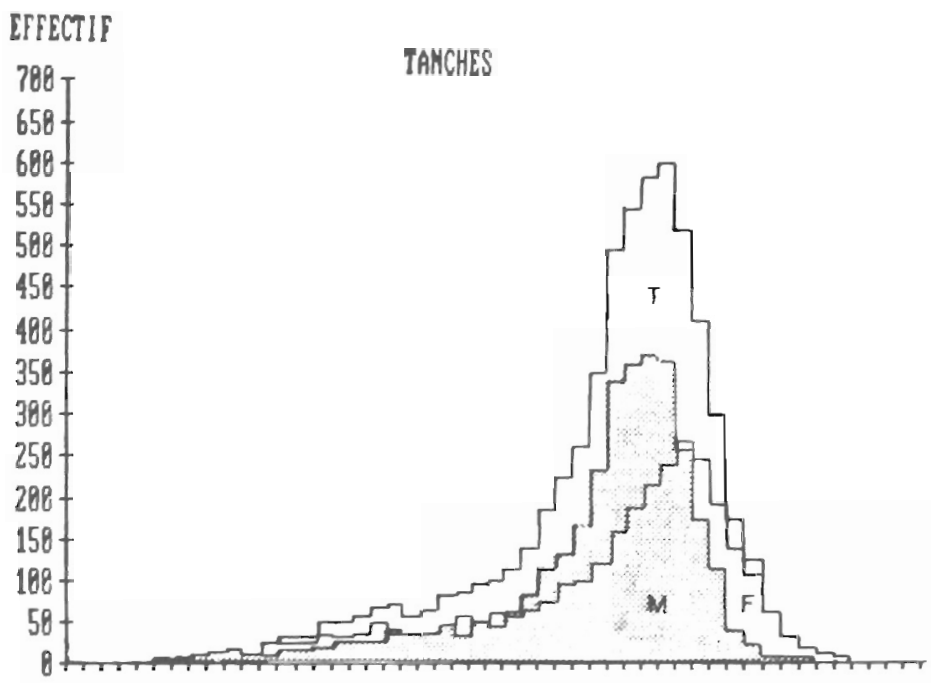

8 B8 118148178298238268298328358386418448478588530 TAlLLE (m)

Fig. 4. - Histogramme des classes de taille pour les tanches $(M=$ mâles, $F=$ femelles; $T=e n-$ semble des tanches).

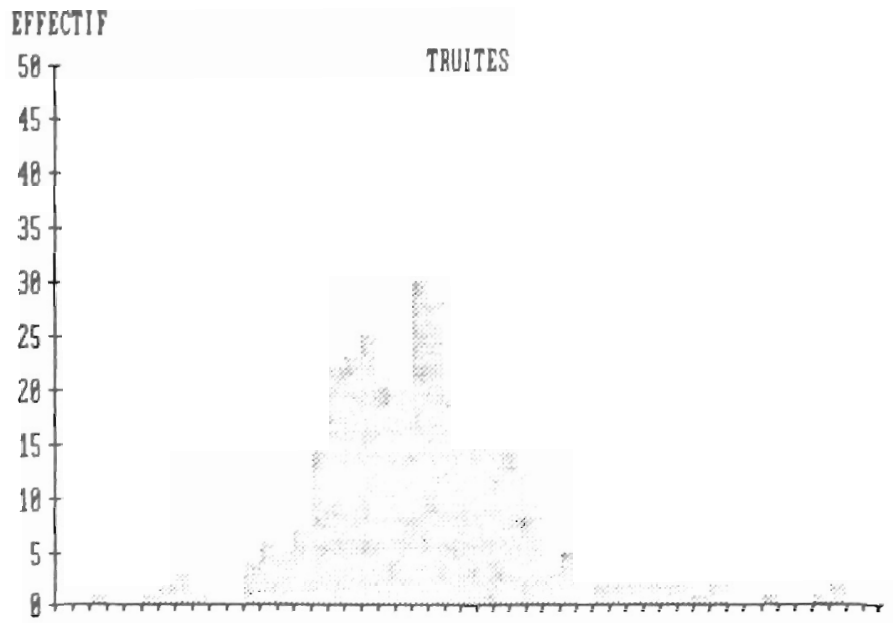

8 $148 \quad 178288230260290328358388418440478580538568598$ TALLLE (m)

Fig. 5. - Histogramme des classes de taille pour les truites. 


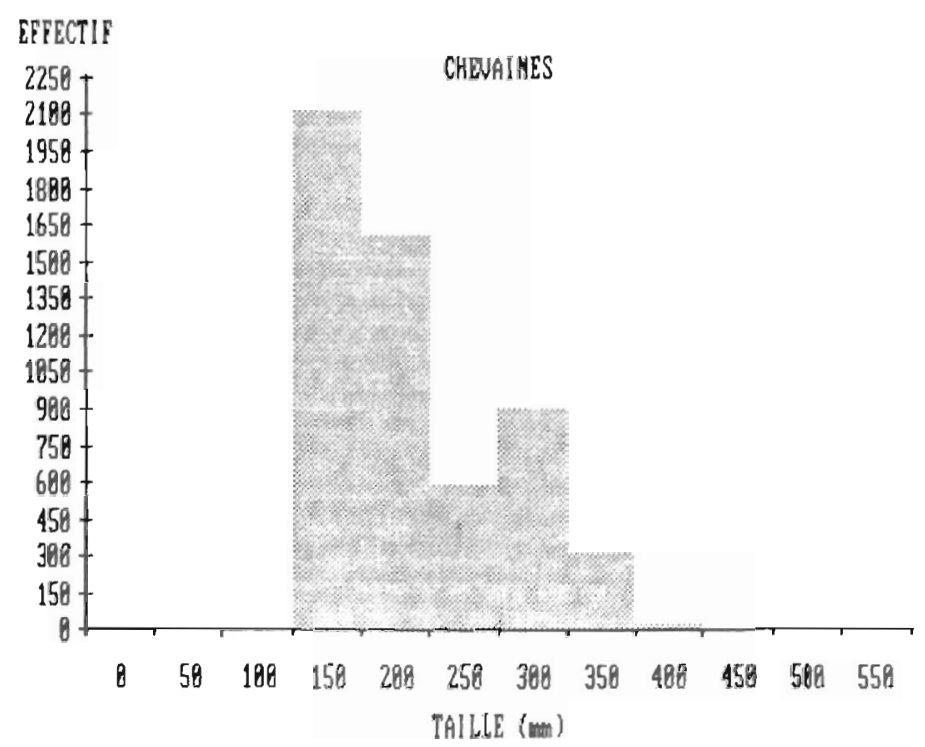

Fig. 6. - Histogramme des classes de taille pour les chevaines.

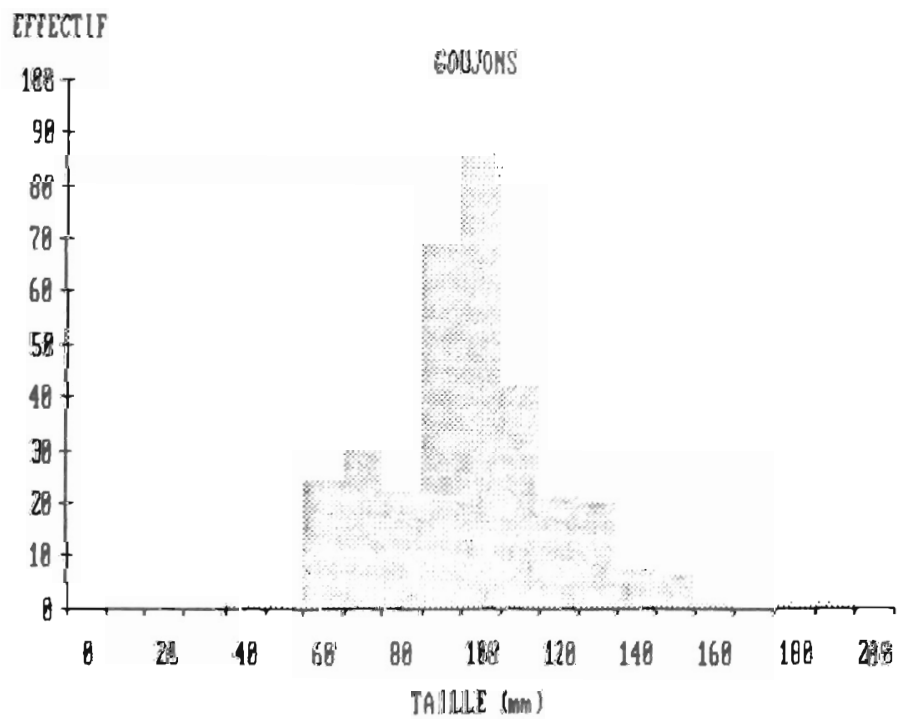

Fig. 7. - Histogramme des classes de taille pour les goujons. 
vidus), les chevaines (Leuciscus cephalus L.) (5 566 individus), les truites (Salmo trutta fario) (342 individus), les goujons (Gobio gobio L.) (332 individus), les gardons (Rutilus rutilus L.) (66 individus), 4 rotengles (Scardinus erythrophtalmus), 1 truite arc-en-ciel (Salmo gairdneri R.) et... 1 carassin (Carassius auratus L.). Ces captures à la pêcherie représentent un total de 12451 individus pour une biomasse de $4661 \mathrm{~kg}$. La répartition par classes de taille des individus appartenant aux principales espèces est représentée dans les figures $4,5,6$ et 7 , respectivement pour les tanches, les truites, les chevaines et les goujons.

La visite de la retenue après vidange a permis de dénombrer 49 tanches (soit environ $35 \mathrm{~kg}$ ), 5 truites pesant $2 \mathrm{~kg}$ et d'évaluer à $40 \mathrm{~kg}$ les chevaines et/ou gardons échoués ou prisonniers de la vase.

A ces résultats il convient d'ajouter les $65 \mathrm{~kg}$ de tanches et les $33 \mathrm{~kg}$ de truites (une centaine d'individus pour chaque espèce) de poissons perdus à la mise en œuvre de la pêcherie).

Pour compléter les estimations, il faut compter les poissons pêchés au filet deux mois auparavant, à l'occasion de pêches pour connaître, préalablement à la vidange, la diversité spécifique et l'abondance relative des espèces de poissons. Ces pêches avaient retiré 120 truites, 192 tanches et 336 chevaines.

Le bilan en nombre et en biomasse, pour les 3 principales espèces, est résumé dans la table $\mathrm{I}$.

\section{DISCUSSION}

\section{Comportement des poissons}

L'expérience de Saints-Peyres a d'abord permis d'observer quelques comportements des poissons lors de la vidange totale de la retenue. Les résultats confirment le comportement rhéophile des poissons qui ne se résolvent à dévaler qu'en état de détresse ou d'affaiblissement. Lors de la vidange de 1974, pour le même site, les poissons avaient commencé à sortir à la cote 622 NGF (Communication EDF-GRPH Languedoc). En 1984, c'est dès la cote 624,80 NGF que les premiers flux de poissons ont été observés en aval mais ce sont des mancuvres exceptionnelles des vannes qui paraissent avoir hâté ce premier flux transitoire (figure 3).

On peut considérer que ce premier passage correspond à des poissons qui stabulaient dans le fond, près de la vanne de prise d'eau qui ont été surpris et entraînés à l'ouverture. Les poissons de ce premier flux sont essentiellement constitués de truites et de tanches. Les truites entrainées alors représentant $21 \%$ de l'effectif de truites de la retenue, on peut considérer que cette espèce s'était rassemblée au fond de la retenue. Par contre les tanches, dont seulement $1,5 \%$ a été entraîné alors, ne semblent pas s'être spécialement accumulées. L'absence de chevaines dans ce premier passage indiquerait que ces poissons préféraient rester en surface ou en queue de retenue. Ces différences de comportement ob- 
servées entre les espèces présentes conditionnent les chances de survie.

L'état des poissons récupérés au premier afflux, était meilleur pour les truites et les tanches que pour les chevaines et goujons. Les poissons qui ont choisi de rester au fond sans manifester de signe d'affolement sont aspirés par l'ouverture subite de la vanne 2 et passent le barrage dans de bonnes conditions. Par contre, les espèces qui sont attirées par les couches d'eau superficielles et celles qui tentent de "remonter" vers la queue de la retenue ne passeront qu'en fin de vidange, dans des conditions très défavorables et après avoir stationné dans des eaux polluées par la remise en suspension des sédiments.

Le flux principal de dévalaison des poissons s'initie lorsque le plan d'eau est à la cote 622 (figure 3), c'est-àdire au même stade qu'en 1974. L'affluence est alors croissante jusqu'en fin de vidange et il est alors difficile de distinguer des différences de comportement entre les espèces qui semblent toutes mélangées, quel que soit l'état physiologique des poissons. Le débit de poissons apparaît alors proportionnel à leur concentration dans le culot de vidange.

En première hypothèse, l'arrivée du flux principal de poissons à la cote 622 pourrait traduire l'existence d'un "espace vital critique" au-dessous duquel les poissons cesseraient de lutter contre la dévalaison; le volume résiduel de la retenue était alors de $100000 \mathrm{~m}^{3}$ et la superficie de 2 ha. En considérant que le nombre de poissons restant dans la retenue s'élevait approximativement à 10000 , cela correspondrait à des densités d'environ 1 poisson pour $10 \mathrm{~m}^{3}$ ou de 1 poisson pour $2 \mathrm{~m}^{2}$. Ces densités n'apparaissent pas en soi élevées et n'expliquent pas à elles seules le déclenchement de la dévalaison, d'autant plus que l'accroissement de densité était progressif tandis que la dévalaison fut plus subite.

La qualité physico-chimique de l'eau (teneur en matières en suspension, en oxygène dissous et en $\mathrm{N} / \mathrm{NH}_{4}{ }^{+}$) a pu jouer un rôle déterminant pour initier la dévalaison. Dans une retenue encaissée comme celle des Saints-Peyres, l'abaissement de la ligne d'eau découvre, en queue de retenue, le lit mineur qui se recreuse rapidement dans la vase. Cette érosion, d'autant plus efficace que la couche de sédiment est épaisse, se fait avec éboulements de blocs de vase dans la rivière. II se forme alors en queue de retenue une masse d'eau chargée qui s'étend vers le barrage. C'est au moment où cette masse d'eau trouble atteint le barrage et apparaît à la sortie des vannes que les poissons perdent tout refuge en eau claire. Le déclenchement de la dévalaison coïncide bien avec l'augmentation de turbidité de l'eau observée au point de vidange. Dans cette hypothèse, il pourrait être suggéré, pour les opérations de vidange ultérieures, de rechercher les conditions hydrauliques qui éviteraient l'extension trop rapide des eaux troubles à toute la retenue et réserveraient une zone d'eau claire pour les poissons. Des mesures seraient cependant à 
entreprendre en d'autres occasions pour suivre la qualité des eaux en divers points de la retenue et juger de la nécessité d'accélérer ou de ralentir les opérations de vidange.

\section{Composition du peuplement}

La composition du peuplement piscicole de la retenue des Saints-Peyres est à cyprinidés dominants, dans une zone qui était pourtant classée en $1^{\text {re }}$ catégorie halieutique. Les truites sont minoritaires par rapport aux cyprinidés (Table I). Les tanches et les chevaines représentent à eux seuls près de $80 \%$ du nombre et de $90 \%$ de la biomasse. Pendant les 10 années de mise en eau depuis la précédente vidange, les gestionnaires de la pêche avaient introduit près de 4 tonnes de truites adultes et de géniteurs réformés et plus de 120000 truitelles dans la retenue des Saints-Peyres, d'ailleurs très fréquentée par les pêcheurs de truites. L'apport des truitelles avait été intense les premières années et avait cessé en 1978, année à partir de la- quelle seules des truites adultes avaient été introduites.

Les truites. Au vu des effectifs restants en 1984, les truites introduites lors des repeuplements ont peu survécu et ont vraisemblablement été pêchées de manière intensive. Les études des classes de taille indiquent un très faible nombre de jeunes truites (figure 5). La quasi-absence de truites de moins de $150 \mathrm{~mm}$, constatée aussi bien lors des pêches préalables que lors de la vidange, indique que le peuplement de truites restant dans la retenue des SaintsPeyres provient presque exclusivement du repeuplement. Sachant que la retenue, par elle-même, ne présente aucune zone propice à la reproduction des salmonidés, les quelques truites de moins de $200 \mathrm{~mm}$ peuvent être considérées comme issues de la rivière amont et des ruisseaux collatéraux.

Les chevaines sont nombreux (5 566 captures à la vidange, table I) et se sont développés naturellement, vraisemblablement à partir de géniteurs présents dans la rivière juste en

Table I. - Evaluation du stock des 3 principales espèces de poissons présentes dans la retenue des Saints-Peyres.

\begin{tabular}{|l|r|r|r|r|r|r|}
\hline & \multicolumn{2}{|c|}{ Tanches } & \multicolumn{2}{c|}{ Chevaines } & \multicolumn{2}{c|}{ Truites } \\
\cline { 2 - 7 } & nombre & $\begin{array}{r}\text { biomasse } \\
(\mathrm{kg})\end{array}$ & nombre & $\begin{array}{r}\text { biomasse } \\
(\mathrm{kg})\end{array}$ & nombre & $\begin{array}{c}\text { biomasse } \\
(\mathrm{kg})\end{array}$ \\
\hline Captures à la vidange & 6149 & 4038 & 5566 & 499 & 342 & 114 \\
Evasions par la grille & 0 & 0 & 1800 & 20 & 0 & 0 \\
Evasions exceptionnelles & 100 & 63 & 0 & 0 & 100 & 33 \\
Echoués dans la retenue & 49 & 35 & $?$ & 40 & 5 & 2 \\
Pêches préalables & 192 & 142 & 335 & 54 & 120 & 42 \\
\hline Total & 6490 & 4278 & 7601 & 613 & 567 & 191 \\
\hline
\end{tabular}


amont qui auraient colonisé la retenue après la remise en eau de 1974. Les classes de taille indiquent deux pics : l'un à $160 \mathrm{~mm}$, pour des individus de 2 ans et l'autre à $280 \mathrm{~mm}$, correspondant à l'âge (déterminé par scalimétrie) de 4 ans. Toutes les classes d'âge sont représentées mais les irrégularités de la structure des tailles traduisent des variabilités interannuelles de la réussite de la reproduction naturelle (figure 6). Cette observation confirme le caractère aléatoire de la reproduction des poissons dans des retenues artificielles soumises à des variations de niveau annuelles et de forte amplitude. Parmi les espèces observées aux SaintsPeyres, le chevaine est la plus ubiquiste et celle qui a une structure de taille la plus complète. Pour évaluer les pertes des individus de petite taille à la grille de la pêcherie, il a été procédé à une rectification du diagramme, par une étude d'extrapolation à partir des classes réellement comptabilisées. Ainsi les individus de la classe $50-100 \mathrm{~mm}$ (entièrement perdus à la grille) sont évalués à 1200 individus soit $9 \mathrm{~kg}$ et les individus de la classe $100-150 \mathrm{~mm}$, dont 2118 individus ont été recueillis a été majorée de $25 \%$ soit de 550 chevaines pesant $11,6 \mathrm{~kg}$. L'estimation des pertes de chevaines à $20,6 \mathrm{~kg}$ et à 1750 individus est concordante avec les observations faites par sondages de pêche électrique.

Les tanches capturées sont au nombre de 6149 (3 213 mâles et 2936 femelles) et représentent $4038 \mathrm{~kg}$. La biomasse des tanches s'élève à $4278 \mathrm{~kg}$ (table II) en te- nant compte des pêches préalables, des pertes à la pêcherie et des individus restés échoués dans la retenue. Les tanches représentent donc $90 \%$ de la biomasse de la retenue des Saints-Peyres. Ce peuplement semble s'être instauré sans intervention humaine, à partir de géniteurs restés dans la rivière à la précédente vidange. Les classements par taille (figure 4) font apparaître un pic unique correspondant à des tailles de $350 \mathrm{~mm}$ à $410 \mathrm{~mm}$ et correspondant à des âges de 8 à 10 ans pour les femelles et 7 à 9 ans pour les mâles. Ce pic de population des tanches résulte d'un recrutement abondant lors des premières années après la remise en eau et qui s'est progressivement dégradé ensuite. La retenue des Saints-Peyres est donc, sitôt après vidange, un milieu de production revalorisée, très propice au développement de la tanche.

\section{Bilan de l'inventaire}

Pour faire ce bilan (table I), on considèrera les poissons effectivement capturés à la pêcherie auxquels s'ajouteront :

- les poissons susceptibles d'avoir passé la grille (valeurs qui ont été estimées, espèce par espèce, au vu des histogrammes par classes de taille);

- les poissons restés échoués dans la retenue (évalués par la visite de contrôle effectuée après vidange);

- les poissons qui ont dévalé avant l'ouverture de la vanne 1 par 
la galerie de dérivation, la nuit du 23 au 24 juillet (il a été déjà mentionné que cette perte résultait d'une manœuvre de vanne imprévue);

- les poissons prélevés lors de pêches d'inventaire préalables, effectuées dans la retenue.

La biomasse totale des poissons, $5090 \mathrm{~kg}$ pour 211 ha soit environ $24 \mathrm{~kg} / \mathrm{ha}$ est faible pour une retenue cyprinicole. Certes la productivité de l'eau est limitée par la minéralisation (bassin versant métamorphique) et par la faiblesse des apports fertilisants liés aux activités humaines. Le vieillissement de la retenue et l'instauration de poissons de grande taille ont aussi contribué à l'abaissement de productivité. Cette retenue pourrait transitoirement produire plus après la période d'assec. En l'absence de gestion, ce sont les tanches qui sont le plus aptes à profiter de ce regain de productivité (comme cela s'est déjà produit 10 ans plus tôt). Si l'on veut instaurer des populations mieux équilibrées, il convient de mettre en œuvre un plan de gestion et de procéder à un repeuplement basé sur des poissons plus jeunes et appartenant à des espèces plus diverses pour mieux utiliser toutes les niches écologiques de la retenue.

\section{REMERCIEMENTS}

Les auteurs tiennent à exprimer leurs sincères remerciements et à dédier cet article à tous ceux qui ont aidé la conception et la réalisation de l'opération de récupération des poissons à la vidange de la retenue des SaintsPeyres : Responsables et Personnels des divers services d'Electricité de France, Membres et Responsables des Associations de Pêche du département du Tarn, chercheurs et Etudiants de l'Ecole Nationale Supérieure Agronomique de Toulouse. La participation de MM. Gras, Vivarelli, Lacroix et Sylvestre a été particulièrement appréciée.

(Travail réalisé avec le financement et la collaboration de Electricité de France : Direction des Etudes et Recherches et Groupe Régional de Production Hydraulique Languedoc).

\section{REFERENCES}

Ackermann W.C., White G.F., Worthington E.8. et Ivens J.L. (eds), 1973. "Manmade lakes : their problems and environmental effects". Am. Geophys. Union, Washington D.C.

Blaxter R.M., 1985. Environmental effects of reservoirs. In "Microbial processes in resenoirs". W. Junk publisher, Dordrecht, chap. 1, pp. 1-26.

Bronte C.R. et Johnson D.W., 1984; Evaluation of the commercial entanglement-gear fishery in Lake Barkley, and Kentucky lake, Kentucky. N. Am. J. Fish. Manag., 4 (1), 75-83.

Cairns J., Benfield E.F. et Webster J.R. (eds), 1978. "Current perspectives on river reservoir ecosystems". N. Am. Benthol. Soc., and Virginia Polytechnic Institute, and State Univ., Blacksburg, Virginia.

George V.C., 1982. Echo location of fishes in Dhudawa reservoir (Madhya Pradesh). Fish. Technol. Soc. Fish. Technol., Cochin, 19 (2), 119-120. 
Gerdeaux D., 1986. Ecologie du Gardon (Rutilus rutilus L.) et du Sandre (Lucioperca lucioperca $L$.) dans le lac de Créteil, de 1977 à 1982 . Etude de la ligulose du gardon. Thèse doct. d'Etat, Université Pierre et Marie Curie, Paris VI, $161 \mathrm{p}$.

Guillard J., 1985. Estimation du stock piscicole du lac Léman, par écho-intégration. DEA Ecologie fond. Appl. eaux continentales, Lyon I, $56 \mathrm{p}$.

Lusk S. et Krcal J., 1983. Fishery exploitation of river valley reservoirs in the Dyje river drainage area. (Tezba ryb z udolnich nadrzi v povodi reky Dyje). Zivocisna Vyroba 28 (11), 809-816.

Mutenia A., 1985. Fish stocks and fishing in the Lokka and Porttipahta reservoirs, northern Finland. In "habitat modification and freshwater fisheries", J.S. Alabaster (ed), Butterworths publisher, Londres, pp. 195-201.

Toran J. et Mermel T.W., 1973. World Register of Dams. Commission internationale des Grands barrages, Paris.

Wondrak P., 1984. Oekologische und fischereiliche Erfahrungen am Ellertshaeuser Strausee. Bayerische Fischereigespraeche Nuernberg 1983, Bayrenth 1984, Wuerzburg. Piwernetz D., Woudrak P. et Klupp R. (ed.), Bayer. Fischereigespraeche. information sr. sporttisch. teichwirte $n^{\circ} 5,111$ 131. 